\title{
Das Bildungserbe der Reformation und seine Bedeutung für heute
}

\section{Reformācijas izglītības mantojums un tā nozīme mūsdienās}

\author{
Friedrich Schweitzer \\ Faculty of Protestant Theology, University of Tübingen, Germany
}

\begin{abstract}
This article refers to the relationship between the Reformation and education. Its special perspective is that of a Protestant heritage which the author identifies and discusses historically and theoretically. In the first part of the article, a brief sketch is given concerning Martin Luther's views of education, with special emphasis on the school and on Religious Education at school as well as on catechetical instruction in church. The second part of the article describes a number of perspectives which could be of importance for today's understanding of education, not only within Protestantism itself but also for a wider public and for the philosophy education. These perspectives refer to, for example, the relationship between faith or religion and education, the relationship between believing and knowing or the anthropology of education. In conclusion, the author identifies a number of new challenges which a Protestant understanding of education has to face up to in today's world. A key challenge which has not been addressed sufficiently in the Protestant tradition is seen in the pluralism of different religions and worldviews characteristic of present-day societies. The author tries to show that there are responses to such challenges which are in line with the Protestant tradition but that this tradition also has to be willing to include new understandings.
\end{abstract}

Keywords: Reformation, education, history of education, philosophy of education.

Die Frage nach der Bedeutung des reformatorischen Bildungserbes heute macht bereits eine erste protestantische Grundentscheidung deutlich, die mir von großer Wichtigkeit scheint. ${ }^{1}$ Es kann auch in der Zeit des 500 jährigen Reformationsjubiläums

1 Dieser Text geht zurück auf einen Vortrag, der am 17. März 2017 in Riga bei der internationalen und interdisziplinären Konferenz "Reformation in der heutigen Welt. 5000 Jahre Reformation” (Universität Latvijas) gehalten wurde. Für den Druck wurde der Text überarbeitet und erweitert, auch in Aufnahme früherer Veröffentlichungen, die in den Vortrag eingegangen waren. Als weiterführende Darstellung vgl. zum Folgenden die monographische Darstellung F. Schweitzer, Das Bildungserbe der Reformation. Bleibender Gehalt, Herausforderungen, Zukunftsperspektiven, Gütersloh, Gütersloher Verlagshaus, 2016. In Abschnitt 1 beziehe ich mich besonders auf F. Schweitzer, Die Reformation als Bildungsbewegung - nicht nur im schulischen Bereich. Ausgangspunkte, Wirkungsgeschichte, Zukunftsbedeutung, in: U. Heckel, J. Kampmann, V. Leppin, C. Schwöbel (Hg.), Luther heute. Ausstrahlungen der Wittenberger Reformation, Tübingen, Mohr Siebeck, 2017, S. 275-293; in Abschnitt 2 habe ich in überarbeiteter Form verschiedene 
nicht unser Ziel sein, das reformatorische Bildungsdenken noch einmal festlich hervorzuheben, um es museal zu bewahren. Das wäre weder pädagogisch hilfreich noch theologisch angemessen. Eine lediglich konservierende Vorgehensweise würde von Anfang an der reformatorischen Einsicht in die Fehlbarkeit widersprechen und zudem auch der Zeitgebundenheit der Reformatoren und ihrer Auffassungen selbst. Mir geht es nicht darum, Luther auf ein Podest zu stellen, um ihn so vermeintlich - zu ehren. Stattdessen nehme ich die durch die Reformation begründete evangelische Bildungstradition in den Blick, um sie auf ihre Zukunftsfähigkeit hin zu befragen. Dabei gehe ich davon aus, dass diese Tradition nicht nur bei Luther, Melanchthon oder Calvin greifbar wird, sondern auch bei späteren protestantischen Bildungstheoretikern wie Comenius oder Schleiermacher sowie in Dokumenten unserer Gegenwart, beispielsweise in kirchlichen Stellungnahmen zu Bildungsfragen. Auf diese Weise soll deutlich werden, dass es um eine lebendige Tradition geht, die sich geschichtlich entfaltet hat und die für die Zukunft offen bleibt. Denn das ist in meiner Sicht das einzig angemessene Verständnis eines "Erbes": Es muss gewiss und an erster Stelle etwas geben, das ein Erbe darstellt, das weitergegeben werden kann. Ein Erbe antreten kann man aber nur, indem man es sich zu eigen macht, es selbst in Gebrauch nimmt und es dabei verwandelt.

Zugleich muss ebenfalls von Anfang an vor Augen stehen, dass es sich unter den heutigen Voraussetzungen keineswegs mehr von selbst versteht, Impulse für das Bildungswesen gerade von Kirche oder Theologie zu erwarten. Hier macht sich ein wesentlicher Unterschied zum 16. Jahrhundert bemerkbar: Kirche und Theologie sind keine selbstverständlichen Experten mehr, denen in Bildungsfragen eine Kompetenz zugetraut würde. Das gilt nicht nur für solche Länder, in denen die Kirchen etwas aufgrund einer sozialistischen Geschichte geschwächt sind - in Osteuropa etwa, aber auch in Ostdeutschland -, sondern es gilt inzwischen für fast alle oder doch die allermeisten Länder. Deshalb soll im Folgenden vor allem auch geklärt werden, in welchem Sinne überhaupt noch von einer über die Kirche selbst hinausreichenden Bedeutung des reformatorischen Bildungserbes heute gesprochen werden kann. Insofern wenden sich die meine Ausführungen bewusst auch an eine breitere Öffentlichkeit sowie an die Erziehungswissenschaft.

Als erstes stellt sich die Frage:

\section{Worin besteht das Bildungserbe der Reformation?}

Ich kann an dieser Stelle keine detaillierte historische Beschreibung geben. Ich beschränke mich auf einige wenige Aspekte, die für das reformatorische

Passagen übernommen aus der ausführlicheren Darstellung F. Schweitzer, Evangelische Impulse für das Bildungswesen - oder: Was bedeutet das reformatorische Bildungserbe heute?, in: C. Spehr (Hg.), Reformation heute. Bd. I: Protestantische Bildungsakzente, Leipzig, Evangelische Verlagsanstalt, 2014, S. 179-194; vgl. weiterhin auch F. Schweitzer, Protestantisches Bildungserbe? Fragen evangelischer Bildungsverantwortung heute, in: Reformationsgeschichtliche Sozietät der Martin-Luther-Universität Halle-Wittenberg (Hg.), Spurenlese. Wirkungen der Reformation auf Wissenschaft und Bildung, Universität und Schule, Leipzig, Evangelische Verlagsanstalt, 2014, 331-347. 
Bildungsdenken kennzeichnend sind. ${ }^{2}$ Dabei werde ich mich besonders an Martin Luther orientieren - nicht weil die Bedeutung Melanchthons, Calvins und anderer Reformatoren im Blick auf Bildungsfragen nicht wichtig wären, sondern aus Gründen des beschränkten Raums. An anderer Stelle habe ich beschrieben, dass sich die Reformation insgesamt auch als eine Bildungsbewegung beschreiben lässt. ${ }^{3}$ Ohne eine Bildungsreform hätte die Reformation ihre Ziele nicht erreichen können. Das gilt nicht nur für Luther, sondern ebenso für andere Reformatoren.

Im Blick auf Luther sind vor allem zwei Schwerpunkte maßgeblich: Bildung in der Schule, einschließlich eines schulischen Religionsunterrichts, einerseits und die katechetische Unterweisung in Kirche und Familie andererseits.

Bemerkenswerterweise wird die Reform des Bildungswesens bereits in der Adelsschrift von 1520 prominent angeführt. Dabei hebt Luther die Notwendigkeit der religiösen Bildung besonders hervor, und zwar in der Gestalt einer biblischen Unterweisung: "Vor allen Dingen sollte in den hohen und niederen Schulen die heilige Schrift die wichtigste und allgemeinste Lektüre sein, und für die jungen Knaben das Evangelium." "Und obwohl die Knaben an erster Stelle genannt werden, bedeutet dies keineswegs, dass die Mädchen ausgeschlossen gewesen wären. Denn Luther fährt fort: "Und wollte Gott, jede Stadt, hätte auch eine Mädchenschule, in der die Mädchen täglich eine Stunde das Evangelium hörten, es wäre deutsch oder lateinisch!"5 Bildungsmöglichkeiten werden also in zwar abgestufter Weise, aber doch eindeutig für beide Geschlechter gefordert.

1520, in den ersten Reformationsjahren, erhält Bildung also bereits einen programmatischen Status. Ihre Konkretion erhält diese Forderung dann später in Luthers sogenannten Schulschriften, mit denen sich Luther zum einen an die Ratsherren und zum anderen an die Eltern wendet. ${ }^{6}$ In beiden Fällen geht es um Begründungen dafür, dass Schulen eingerichtet und unterhalten werden sollen sowie Eltern die Pflicht haben, ihre Kinder zur Schule zu schicken - eine Forderung, die deshalb so wichtig war, weil es so etwas wie eine allgemeine Schulpflicht damals noch nicht gab (Luther war einer der ersten, der sich für eine solche Schulpflicht aussprach, weshalb er in der Geschichte der Volksbildung oder auch der Volksschule

2 Weiterführende Hinweise auch auf Literatur finden sich in den in Anmerkung 1 angegebenen Veröffentlichungen.

3 Vgl. F. Schweitzer, Die Reformation als Bildungsbewegung - nicht nur im schulischen Bereich. Ausgangspunkte, Wirkungsgeschichte, Zukunftsbedeutung, in: U. Heckel, J. Kampmann, V. Leppin, C. Schwöbel (Hg.), Luther heute. Ausstrahlungen der Wittenberger Reformation, Tübingen, Mohr Siebeck, 2017, S. 275-293.

4 M. Luther, An den christlichen Adel deutscher Nation: Von des christlichen Standes Besserung, in: K. Bornkamm, G. Ebeling (Hg.), Martin Luther, Ausgewählte Schriften, Bd. 1, Frankfurt/M., Insel, ${ }^{2} 1982$, S. 150-237, 230.

Ibid.

6 M. Luther, An die Ratsherrn aller Städte deutschen Landes, dass sie christliche Schulen aufrichten und halten sollen, in: K. Bornkamm, G. Ebeling (Hg.), Martin Luther, Ausgewählte Schriften, Bd. 5, Frankfurt/M., Insel, ${ }^{2} 1982$, S. 40-72; ders., Eine Predigt Martin Luthers, dass man Kinder zur Schule halten solle, in: ibid., S. 90-139. Auf diesen beiden Quellen beruhen die Ausführungen im Folgenden. 
eine wichtige Rolle spielt ${ }^{7}$ ). Darüber hinaus wird aber auch genauer beschrieben, was Schulen leisten sollen und was dort zu lehren sei.

Für Luthers Argumentation bezeichnend ist eine Argumentation, die jeweils nach zwei Seiten hin entfaltet wird. Schulen haben für ihn eine "geistliche" und eine "weltliche" Bedeutung. Diese Argumentationsweise geht natürlich auf Luthers sogenannte Zwei-Reiche-Lehre zurück. In der Geschichte der Pädagogik eröffnete die weltliche Betrachtungsweise eine neue Möglichkeit, sich nicht nur theologisch, sondern - wie es heute heißt - erziehungswissenschaftlich mit Schule und Bildungsfragen zu befassen. Darin kann - in heutiger Einschätzung ein pädagogischer Rationalitätsgewinn gesehen werden, weil Bildung nun auch unabhängig von Erlösung thematisiert werden kann, und zugleich ein historischer Grund dafür, warum die wissenschaftliche Pädagogik in Deutschland stark protestantisch ausgerichtet war, abzulesen an Klassikern wie Johann Amos Comenius, Johann Gottfried Herder, Johann Heinrich Pestalozzi, Friedrich Schleiermacher und Johann Friedrich Herbart oder, im 20. Jahrhundert, Eduard Spranger und Wilhelm Flitner, die allesamt dem Protestantismus angehörten. ${ }^{8}$

Seine Begründung der Notwendigkeit von Schule und Bildung führt Luther im Einzelnen zunächst in geistlicher Hinsicht aus. Konkret geht es ihm dabei um die Vorbereitung für das Predigtamt, das ja seinerseits das Zentrum eines der wichtigsten reformatorischen Anliegen war. Denn der Gottesdienst und insbesondere die Predigt sollte nun in der Muttersprache gehalten werden. Deshalb änderten sich auch die Anforderungen an die Pfarrer, die nun nicht mehr bloß einen lateinischen Text verlesen können mussten, den weithin vielleicht niemand von den Gemeindegliedern verstand. Jetzt brauchte man in der Kirche Menschen, die selbst die Bibel auslegen und die selbständig in der Muttersprache eine Predigt verfassen können. Das erklärt, warum im Zentrum des auf die geistliche Seite bezogenen reformatorischen Bildungsdenkens vor allem die alten Sprachen stehen mussten - Griechisch und Hebräisch sowie Latein -, auch wenn Luther durchaus auch andere Bildungsinhalte wie etwa Geschichte empfiehlt. Und auch wenn der "geistliche Nutzen" von Bildung hier im Vordergrund stand, kritisierte Luther die vorreformatorisch einflussreichen Klosterschulen gerade deshalb, weil man in ihnen nur Singen und Beten geübt habe. Zumindest in diesem Sinne ging es Luther nicht um eine verkirchlichte Schule. Eher folgte er den Anliegen des damals aufstrebenden Humanismus, eben mit der Akzentuierung der sprachlichen Bildung.

Auf der einen Seite dienen Bildung und Schule nach Luther also klar der kirchlichen Verkündigung. Auf der anderen Seite steht ihr "weltlicher Nutzen", den Luther nicht weniger eindringlich, wenn auch mit anderen Argumenten herausarbeitet und hervorhebt. Bedeutsam ist dafür die übergreifende Zielsetzung, die Luther der schulischen Bildung im weltlichen Sinne vorschreibt. Neben der Vorbildung für das Pfarramt soll die Schule "zeitlichen und vergänglichen Frieden,

7 Vgl. etwa W. Flitner, Die vier Quellen des Volksschulgedankens, Hamburg: Hansischer Gildenverlag, 1941.

8 Vgl. dazu F. Schweitzer, Pädagogik und Religion. Eine Einführung, Stuttgart, Hohlhammer, 2003; ders., Bildung, Neukirchen-Vluyn, Neukirchener, 2014. 
Recht und Leben" garantieren. Gemäß der lutherischen Theologie lässt sich auch sagen, dass Bildung für beide Reiche oder Regimente zentral ist - für das geistliche Regiment nicht weniger als für das weltliche Regiment. Im Einzelnen geht es dann um verschiedene Ämter oder Berufe, die ohne Bildung nicht versehen oder ausgeübt werden können. Genannt werden beispielsweise Schulmeister und Schreiber. Besonders hervorgehoben werden darüber hinaus die Juristen, was auch historisch und politisch aufschlussreich ist. Luther trat ein für eine gesellschaftliche Ordnung, die anders als die mittelalterliche Fehdeordnung nunmehr auf geschriebenem Recht beruhen sollte. Das erklärt, warum gebildete Juristen in seiner Sicht unerlässlich waren. Und Luthers Argumentation geht sogar noch einen Schritt weiter: Auch Regierungstätigkeiten sollten an Bildungsvoraussetzungen oder eine entsprechende Qualifikation und also nicht mehr allein an die adlige Herkunft gebunden sein, was fast demokratisch anmutet, auch wenn demokratische Zielsetzungen damals noch keine Rolle spielten. ${ }^{9}$

Bei seinem Bildungsdenken hatte Luther aber nicht nur die Schule im Blick. Vielmehr bezieht er sich auch auf die Familie und auf die Kirche bzw. die Gemeinde. Hier war es dann der katechetische Unterricht, der von Luther gefordert und unterstützt wurde, vor allem mit seinen Katechismen, aber auch ganz allgemein mit der Forderung nach religiöser bzw. christlicher Unterweisung für alle. ${ }^{10}$ Ziel war ein verstehender Glaube - Christen sollen nicht nur etwas auswendig können, sondern sie sollen auch verstehen, was der christliche Glaube bedeutet. Ziel war aber auch eine urteilsfähige Gemeinde. Die Gemeinde, so Luther, soll das auf der Kanzel Vorgetragene beurteilen können. ${ }^{11}$ Das entsprach dem von Luther vertretenen Prinzip des "Priestertums aller Gläubigen". Weiterhin erwächst das katechetische Anliegen aus dem reformatorischen Glaubensverständnis und aus der Rechtfertigungslehre: Wenn jeder und jede Einzelne in einem unmittelbaren Verhältnis zu Gott steht und es allein auf den persönlichen, unvertretbaren Glauben ankommt, dann reicht es nicht aus, wenn nur der Klerus sich auf Glaubensfragen versteht. Dass auch im Blick auf die katechetische Unterweisung von Bildung gesprochen werden kann, findet seine Begründung meines Erachtens in der Betonung des Verstehens, das nun jeder Christ erreichen sollte. Das bedeutet nicht, dass das reformatorische Bildungsverständnis einfach mit dem sogenannten klassischen Verständnis von Bildung gleichgesetzt werden könnte, wie es sich dann im 18. und 19. Jahrhundert entwickelt hat. Eine

9 Vgl. M. Luther, Eine Predigt Martin Luthers, dass man Kinder zur Schule halten solle, in: : K. Bornkamm, G. Ebeling (Hg.), Martin Luther, Ausgewählte Schriften, Bd. 5, Frankfurt/M., Insel, ${ }^{2} 1982$, S. 90-139, 131.

${ }^{10}$ Vgl. bes. M. Luther, Der Kleine Katechismus. In: Die Bekenntnisschriften der evangelisch-lutherischen Kirche, hg. im Gedenkjahr der Augsburgischen Konfession 1530, Göttingen, Vandenhoeck \& Ruprecht, ${ }^{7} 1976$, 507-527; pädagogisch interessant ist insbesondere auch die Vorrede.

11 Vgl. M. Luther, Dass eine christliche Versammlung oder Gemeinde Recht und Macht habe, alle Lehre zu beurteilen und Lehrer zu berufen, ein- und abzusetzen, Grund und Ursache aus der Schrift, in: K. Bornkamm, G. Ebeling (Hg.), Martin Luther, Ausgewählte Schriften, Bd. 5, Frankfurt/M., Insel, ${ }^{2} 1982$, S. 7-18. 
Kontinuität, die die Anwendung des Bildungsbegriffs auch im Blick auf das 16. Jahrhundert und die Reformation erlaubt, ist aber doch erkennbar gegeben.

Beide Reformanliegen zusammen, also für Schule und für Kirche oder Gemeinde, haben dazu geführt, dass die Reformation zu einer Bildungsbewegung geworden ist. Und wenn oben festgehalten wurde, dass die Reformation ohne Bildung nicht hätte gelingen können, so kann dem nun hinzugefügt werden, dass die Reformation zugleich auch ein wirksames Motiv für Bildung war. Denn die evangelischen Christen entwickelten ein neues Interesse an einem verständigen Glauben, das ohne Bildung nicht zu erreichen war.

Damit ist - in freilich äußerst knapper Form - skizziert, worin das reformatorische Bildungserbe bestehen kann. Die Reformation war auch eine Bildungsbewegung, was all diejenigen, die sich heute auf die Reformation berufen, auch zu einem entsprechenden Bildungsengagement verpflichtet. Es gilt aber auch weiterhin, dass die Bezeichnung als Erbe eine zeitgenössische und damit aktualisierende Rezeption einschließen muss. Darum soll es im Folgenden gehen. Auf die Wirkungsgeschichte hingegen kann hier nicht eigens eingegangen werden. ${ }^{12}$

\section{Was kann das reformatorische Bildungserbe für unsere Gegenwart bedeuten?}

Es ist von vornherein davon auszugehen, dass sich eine Bildungsbewegung, die im Spätmittelalter bzw. in der frühen Neuzeit entstanden ist, mit ihren damaligen Anliegen, Zielen und praktischen Maßnahmen sicher nicht einfach auf unsere Gegenwart übertragen lässt. Das wäre ein völlig unhistorisches Vorgehen, das an den veränderten gesellschaftlichen, politischen, kulturellen und kirchlichen Voraussetzungen unserer Zeit vorbeiginge. Statt dessen kommt es nun darauf an, Perspektiven zu entwickeln, die einerseits im Sinne des Bildungserbes zentrale Bildungsanliegen der Reformation aufnehmen, die aber andererseits ihre Plausibilität im Blick auf die Gegenwart gewinnen. Die im Folgenden in Gestalt von vier knappen Thesen entwickelte Antwort auf die Frage nach der Gegenwartsbedeutung des reformatorischen Bildungsverständnisses folgt diesem Anliegen. ${ }^{13}$

12 Vgl. dazu Schweitzer, Das Bildungserbe der Reformation sowie K. E. Nipkow, F. Schweitzer (Hg.), Religionspädagogik. Texte zur evangelischen Erziehungs- und Bildungsverantwortung seit der Reformation. 3 Bde., München, Kaiser, 1991 und Gütersloh, Gütersloher Verlagshaus, 1994. Als systematische Darstellung vgl. auch R. Preul, Evangelische Bildungstheorie, Leipzig, Evangelische Verlagsanstalt, 2013.

${ }^{13}$ Die Thesen beruhen auf meiner Sicht evangelischer Bildungsverantwortung, wie ich sie $\mathrm{m}$ Blick auf die Reformation und das reformatorische Bildungserbe in den in Anmerkung 1 genannten Veröffentlichungen entwickelt habe. Sie berühren darüber hinaus eine breite pädagogische und theologische Diskussion, die sich jedoch in Gestalt von Einzelanmerkungen kaum einfangen lässt. Vgl. auch F. Schweitzer, Religionspädagogik, Gütersloh, Gütersloher Verlagshaus, 2006. 


\subsection{Evangelisches Bildungsengagement muss heute als Form demokratischer Bildungsmitverantwortung wahrgenommen werden!}

Bekanntlich ist das staatliche Bildungswesen mit der Trennung von Staat und Kirche aus dem unmittelbaren Verantwortungsbereich der Kirche ausgetreten. In den allermeisten Staaten ist die Schule heute eine rein staatliche Einrichtung. Eine direkte Form der Mitgestaltung der Schule durch die Kirchen gibt es deshalb nicht mehr, zumindest abgesehen vom schulischen Religionsunterricht, bei dem es kirchliche Mitwirkungsrechte geben kann. Auf wissenschaftlicher Ebene spiegelt sich die Trennung zwischen Schule und Kirche schon seit Jahrzehnten in der Forderung nach einer autonomen Pädagogik oder Erziehungswissenschaft, die sich inzwischen auch weithin durchgesetzt hat. Pädagogische Autonomie zielt nicht zuletzt auf die Unabhängigkeit der Erziehungswissenschaft von theologischen und kirchlichen Voraussetzungen oder Einflussnahmen. Die Erziehungswissenschaft der Gegenwart will in religiöser und weltanschaulicher Hinsicht neutral sein - ein Anliegen, das zwar auch kritisch zu diskutieren, an dieser Stelle aber zunächst als Faktum wahrzunehmen ist. ${ }^{14}$ Im letzten Teil des Beitrags komme ich noch einmal darauf zurück.

Unter diesen Voraussetzungen scheint für evangelische Impulse für das Bildungswesen wenig Raum zu sein. Es gibt inzwischen aber auch gegenläufige Entwicklungen, die im vorliegenden Zusammenhang besonders interessant sind. In diesem Sinne kann vor allem auf die Anforderungen demokratischer Entscheidungsfindung verwiesen werden. Das Bildungswesen ist in dieser Sicht nicht einfach eine Angelegenheit hoheitlich-staatlicher Entscheidungsautonomie, sondern muss, im Sinne einer Demokratisierung der Lebensverhältnisse, mit gesellschaftlichen Formen der Mitverantwortung verbunden werden. Dafür liefert der Protestantismus bereits in der Reformationszeit ein Vorbild, das heute am angemessensten als eine Form zivilgesellschaftlicher Mitverantwortung für das Bildungswesen beschrieben werden kann.

Zur demokratischen Ausgestaltung des Bildungswesens kann die Kirche dabei noch in einer weiteren wesentlichen Hinsicht beitragen. Zur Demokratie gehört auch ein Trägerpluralismus, d.h. es soll nicht nur staatliche Bildungseinrichtungen geben, weil sonst ein staatliches Bildungsmonopol droht - als Gefährdung von Demokratie, wie der Erfahrung in totalitär verfassten Staaten deutlich zu entnehmen ist. ${ }^{15}$ Indem die Kirche Bildungsangebote unterhält und als Träger eigener, also evangelischer Bildungseinrichtungen tätig ist, trägt sie bei zur Garantie eines pluralistischen Bildungswesens.

\subsection{Evangelische Bildung muss das Warum und Wozu zum Thema machen!}

Die Frage nach dem Warum und Wozu liegt gleichsam bewusst quer zur gegenwärtigen Bildungsdiskussion. Der Bildungsdiskurs im 21. Jahrhundert steht

${ }^{14}$ Stellvertretend genannt sei W. Brezinka, Von der Pädagogik zur Erziehungswissenschaft. Eine Einführung in die Metatheorie der Erziehung, Weinheim, Beltz, 1971.

${ }^{15}$ Vgl. dazu grundlegend P. Häberle, Erziehungsziele und Orientierungswerte im Verfassungsstaat, Freiburg, München, Alber, 1981. 
bislang ganz im Zeichen der empirischen Bildungsforschung und der Kompetenzen, deren Erwerb und Ausprägung etwa bei Vergleichsuntersuchungen im Zentrum stehen. Seit den großen internationalen Schulleistungsvergleichs untersuchungen, die ihr Symbol in den PISA-Studien gefunden haben, wird mit großer Intensität an der Entwicklung von Bildungsstandards gearbeitet. All dies dient erkennbar dem Wunsch nach mehr Effektivität und Nachhaltigkeit im Bildungswesen und ist insofern auch aus evangelischer Sicht zu begrüßen. Das Bildungswesen soll leisten, was es verspricht - und das entscheidende Kriterium dafür kann kein anderes sein als der tatsächliche Lernerfolg der Schülerinnen und Schüler.

Allerdings bleibt auch dann noch eine zentrale Frage offen: Was sind die Ziele, auf die mit gesteigerter Effektivität hingearbeitet werden soll? Und wie lassen sich diese Ziele begründen? Aufschlussreich sind hier die Darstellungen der OECD, die hinter den PISA-Studien stehen. Der Bericht der OECD "Knowledge and Skills for Life" kommt ganz ohne bildungstheoretische Ausführungen aus, jedoch nicht ohne ein Menschenbild. Die Zugangsweise von PISA rechtfertige sich, so wird hier gesagt, durch den "Erfolg im Leben" ("to succeed in life"). Aber was heißt Erfolg? Die Antwort ist kurz und bündig: "labour market success and earnings". Oder noch einmal zugespitzt: "better jobs" und "higher salaries". ${ }^{16}$

Die Aufgabe für eine evangelische Bildungsmitverantwortung kann hier nur darin bestehen, die Frage nach dem Warum und Wozu von Bildung und Schule wach zu halten. Die Antwort - Geld und Karriere - stellt gerade keine überzeugende Antwort auf diese Frage dar, weder bildungstheoretisch noch theologisch. Das wird nicht zuletzt an den jeweils leitenden Menschenbildern deutlich, worauf sich deshalb auch die nächste These eigens bezieht.

\subsection{Das evangelische Bildungsverständnis muss die Frage nach dem Menschenbild der Bildung wach halten!}

Die Frage nach dem Menschenbild, das hinter dem Bildungsverständnis steht, gilt heute vielen als wenig zeitgemäß. ${ }^{17}$ Solche allgemeinen Fragen lenken demnach ab von den faktisch-praktischen Problemen im Bildungswesen, wie sie in der beschriebenen Effektivitätsperspektive doch viel deutlicher zum Ausdruck kämen. Und viele praktische Herausforderungen hätten am Ende doch reichlich wenig mit abstrakten philosophisch-anthropologischen Bestimmungen zu tun.

Solche Einwände sind auch aus meiner Sicht durchaus berechtigt, sofern Bildungsfragen allein auf der Ebene von Menschenbildern geklärt werden sollen, was in der Vergangenheit mitunter durchaus der Fall war. Die Kritik an der Menschenbildfrage wird aber falsch und führt sogar in die Irre, wenn sie eine grundsätzliche Irrelevanz aller Fragen dieser Art behaupten will.

${ }^{16}$ OECD, Knowledge and skills for life. First Results from the OECD Programme for International Student As-sessment (PISA) 2000, Danvers, OECD, 2001, S. 19f.

${ }^{17}$ Vgl. etwa den in dieser Hinsicht bewusst zurückhaltenden Zugang bei H.-E. Tenorth, "Alle alles zu lehren”. Möglichkeiten und Perspektiven allgemeiner Bildung, Darmstadt, WBG, 1994. 
Geld und Karriere, so haben wir gesehen, reichen hier als Antwort nicht zu. Deshalb erinnere ich an dieser Stelle noch einmal an die Zielformulierungen der Reformation: Alle Bildung soll einem Leben in Frieden und Gerechtigkeit dienen. Heute wird in dieser Hinsicht von Bildungsgerechtigkeit gesprochen sowie davon, dass eine solche Gerechtigkeit immer als Befähigungsgerechtigkeit verstanden werden muss. ${ }^{18}$ Gerechtigkeit im Bildungswesen ist dann nur so zu erreichen, dass jeder und jede einzelne zur Teilhabe am gesellschaftlichen Leben befähigt wird. Genau so lässt sich später auch beispielsweise das berühmte Programm des Comenius aus dem 17. Jahrhundert verstehen: allen alles gründlich zu lehren. ${ }^{19}$ Bei Comenius wird dies auch noch einmal deutlicher als im 16. Jahrhundert auf alle Menschen ohne Unterschied bezogen: "Wo Gott keinen Unterschied gemacht hat, da soll auch der Mensch keine Schranken aufrichten." ${ }^{20}$ Bildungsmöglichkeiten müssen allen offen stehen - und alle müssen dazu befähigt werden, sich am gesellschaftlichen Leben zu beteiligen. Bemerkenswert ist dabei, dass Comenius nun schöpfungstheologisch argumentiert und auf diese Weise eine Grundlegung für seine damals durchaus revolutionäre pädagogische Ausrichtung gewinnt: Nicht mehr die soziale Herkunft oder Zugehörigkeit soll über den Zugang zu Bildungsmöglichkeiten entscheiden, sondern tatsächlich allen Menschen eignet als Geschöpfen Gottes ein Recht auf Bildung, wie dies modern ausgedrückt werden muss. So klar war dies im 16. Jahrhundert noch nicht formuliert worden. Heute ist es zu einem Grundanliegen protestantischen Bildungsdenkens geworden. ${ }^{21}$

Dahinter steht eine Grundoption, die in unserer Gegenwart etwa in der ebenfalls grundlegenden Bildungsdenkschrift der Evangelischen Kirche in Deutschland aus dem Jahr 2003 schon im Titel zum Ausdruck gebracht wird: "Maße des Menschlichen" der Mensch, hier verstanden in seiner Geschöpflichkeit und Gottebenbildlichkeit, dadurch ausgestattet mit einer von Gott geschenkten und deshalb unveräußerlichen Würde, soll das Maß der Bildung sein. ${ }^{22}$ Ein solches Menschenbild widerspricht jedem Versuch, Bildung beispielsweise allein an ökonomischen Erfordernissen, an Karriere und Einkommen zu bemessen.

Mit der heute für das evangelische Bildungsdenken so bedeutsamen Berufung auf die Gottebenbildlichkeit als Grundlage der Menschenwürde sowie, damit verbunden, auch auf das Recht des Kindes als Ausgangspunkt aller Pädagogik und Bildung, kommt es freilich auch zu einer zumindest teilweisen Verschiebung, die

${ }^{18}$ Vgl. dazu M. Nussbaum, Frontiers of justice: disability, nationality, species, Cambridge, Mass., Belknap Press, 2006; zur Rezeption im vorliegenden Kontext s. F. Schweitzer, Menschenwürde und Bildung. Religiöse Voraussetzungen der Pädagogik in evangelischer Perspektive, Zürich, TVZ, 2011.

19 Vgl. J.A. Comenius, Pampaedia. Lateinischer Text und deutsche Übersetzung, Heidelberg, Quelle \& Meyer, ${ }^{2}$ 1965; dieses grundlegende Werk ist in der Einleitung nach den drei genannten Begriffen bzw. Kategorien aufgebaut (omnes, omnia, omnono).

${ }^{20}$ Comenius, Pampaedia, S. 31.

${ }^{21}$ Vgl. Schweitzer, Menschenwürde.

22 Vgl. Evangelische Kirche in Deutschland, Maße des Menschlichen. Evangelische Perspektiven zur Bildung in der Wissens- und Lerngesellschaft. Eine Denkschrift, Gütersloh, Gütersloher Verlagshaus, 2003. 
für das Verständnis des Begriffs Bildungserbe aufschlussreich ist. Denn beides, die Menschenwürde und das Recht des Kindes, wurde im 16. Jahrhundert gerade noch nicht als Ausgangspunkt gewählt. Im Rückblick können wir solche Motive in der reformatorischen Theologie zwar angelegt sehen, aber es kann doch keinen Zweifel daran geben, dass der Protestantismus in seiner Geschichte entwicklungsoffen war und sein musste, wenn er in seinem Bildungsverständnis plausibel bleiben wollte, und dass er sich auch tatsächlich als lernfähig erwiesen hat. Immer wieder wurden und werden evangelische Motive nicht einfach als festliegende kirchliche Dogmatik bewahrt, sondern diese Motive sind von einem freien Protestantismus und einer protestantischen Kultur aufgenommen und zur Geltung gebracht worden, manchmal auch gegen die evangelische Kirche.

Aus meiner Sicht ist dies auch der Punkt, an dem das Verhältnis zwischen Erziehungswissenschaft und Religion oder Weltanschauung heute noch einmal neu zur Diskussion gestellt werden muss. Um es wenigstens in Gestalt einer These anzudeuten: Die Erziehungswissenschaft in der westlichen Welt lebt von religiösen Voraussetzungen, die heute nur noch selten als solche erkannt werden, die sich aber gerade nicht von selbst verstehen. ${ }^{23}$ Insofern ist die oben angesprochene Entwicklung, in deren Folge sich die Erziehungswissenschaft der Gegenwart allein auf eine religiös und weltanschaulich neutrale Grundlegung beruft, nicht ausreichend. Sie übergeht, dass in der Pädagogik nicht einfach auf alle normativen Festlegungen verzichtet werden kann, was in übergreifenden normativen Orientierungen der Erziehungswissenschaft wie der Berufung auf das Kindeswohl durchaus auch noch präsent ist. Solche Orientierungen bedürfen aber der Konkretion, was ohne Aufnahme weiterer normativer Bestimmungen kaum möglich ist.

\subsection{Das evangelische Bildungsverständnis muss den Zusammenhang von Bildung und Glaube stärken!}

In evangelischer Sicht - und das ist heute besonders herausfordernd besteht zwischen Bildung und Glaube ein unauflöslicher Zusammenhang. Dieser Zusammenhang ist dabei keineswegs nur für die Kirche bedeutsam, sondern gerade auch für Gesellschaft und Öffentlichkeit. Dabei ist dieser Zusammenhang nach beiden Seiten zu lesen: Keine Bildung ohne Glaube oder Religion - kein Glaube ohne Bildung.

\section{Zunächst zu der Forderung: Keine Bildung ohne Glaube oder Religion!}

Für die Reformatoren stand von Anfang an fest, dass der Glaube und namentlich die Bibel eine zentrale Stellung im Bildungswesen haben müssen. Im Anschluss an die oben beschriebenen Forderungen Luthers in der Adelsschrift schon von 1520 lässt sich dies, wie im ersten Teil meiner Darstellung gezeigt, so formulieren, dass das, was die menschliche Existenz letztlich trägt, in der Schule nicht ausgeblendet bleiben darf. Für Luther war das das Evangelium - allgemeiner würden wir heute von Religion oder Lebenssinn sprechen. Kinder und Jugendliche brauchen dabei eine kompetente Begleitung, etwa durch den Religionsunterricht.

${ }^{23}$ Vgl. Schweitzer, Pädagogik und Religion. 
Bildung kann nicht vollständig sein und ihrer Aufgabe nicht gerecht werden, wenn sie diese Dimension aussparen wollte.

Bei der Forderung: Keine Bildung ohne Religion geht es konkret auch darum, dass der Mensch und eine menschengerechte Bildung nicht auf die materielle Seite beschränkt werden dürfen. Wer dies, etwa im Namen eines ökonomischen Utilitarismus, versucht, so etwa Friedrich Schleiermacher schon vor 200 Jahren in seinen berühmten "Reden über die Religion", der bringt Kinder unfehlbar um ihren Sinn. ${ }^{24}$

Keine Bildung ohne Religion - das bedeutet aber auch, dass es zur Bildung gehört, ein kritisch-reflektiertes Verhältnis zum eigenen Glauben zu gewinnen. Die durch Bildung entwickelte Vernunft soll vor dem Glauben nicht Halt machen, und sie soll nicht als Alternative zum Glauben missverstanden werden. Stattdessen geht es in evangelischer Sicht um eine Verbindung von Glaube und kritischer Vernunft, im Sinne eines spannungsvollen, am Ende aber doch komplementären Verhältnisses. Das Ziel einer solchen Bildung heißt dann nicht: Mündigkeit statt Glaube, sondern religiöse Mündigkeit als eigene Urteilsfähigkeit im Glauben, wie im deutlichen Anschluss an Luther ebenfalls Schleiermacher formuliert. ${ }^{25}$

Der Forderung: Keine Bildung ohne Religion entspricht deshalb auf der anderen Seite das komplementäre Postulat: Kein Glaube ohne Bildung! Auch dafür sind die Reformatoren von Anfang an eingetreten. Religiöse Bildung - das Verstehen des Glaubens - sollte nicht länger auf den kleinen Kreis der Kleriker begrenzt sein. Alle Kinder und Jugendlichen, aber auch alle Erwachsenen sollten vielmehr durch die katechetische Unterweisung erreicht werden. Und Luther setzte sich mit großer Konsequenz ausdrücklich immer wieder dafür ein, dass diese Unterweisung auf "Kinder und Einfältige" ausgerichtet sein sollte, also auf Menschen, die noch nicht gebildet sind.

Auch von dieser Seite her, vom Glauben her betrachtet, kann also von einer sinnvollen Verbindung zwischen Glaube und Vernunft gesprochen werden. Ein eindrückliches Beispiel dafür ist heute das spannungsvolle Verhältnis zwischen Schöpfungsglaube und Evolutionstheorie. ${ }^{26}$ Anders als der sogenannte neue Atheismus behauptet, steht der biblische Schöpfungsglaube gerade nicht in einem Gegensatz zu naturwissenschaftlichen Erklärungsmöglichkeiten. ${ }^{27}$ Der Schöpfungsglaube will keine naturwissenschaftlichen Erklärungen bieten, so wie umgekehrt naturwissenschaftliche Erklärungsmodelle keine Aussagen über den

${ }^{24}$ Vgl. F. Schleiermacher, Über die Religion. Reden an die Gebildeten unter ihren Verächtern (1799), hg. v. R. Otto, Göttingen, Vandenhoeck \& Ruprecht, ${ }^{6} 1967$, dritte Rede.

${ }^{25}$ Vgl. F. Schleiermacher, Die praktische Theologie nach den Grundsätzen der evangelischen Kirche im Zusammenhange dargestellt, hg. v. J. Frerichs, Berlin, Reimer, 1850, S. 396.

${ }^{26}$ Vgl. dazu Weltentstehung, Evolutionstheorie und Schöpfungslaube in der Schule. Eine Orientierungshilfe des Rates der Evangelischen Kirche in Deutschland, Hannover, EKD, 2008; F. Schweitzer, Schöpfungsglaube - nur für Kinder? Zum Streit zwischen Schöpfungsglaube, Evolutionstheorie und Kreationismus, Neukirchen-Vluyn, Neukirchener Verlag, 2012.

27 Gemeint ist etwa R. Dawkins, Der Gotteswahn, Berlin, Ullstein, 2008. 
Sinn des Lebens oder gar über Gott treffen können. Auch in diesem Falle ist die Vorstellung einer Komplementarität von Schöpfungsglaube und Naturwissenschaft hilfreich. ${ }^{28}$ Beide können einander nicht ersetzen, und beide bleiben doch auf Dauer sinnvoll.

Es versteht sich von selbst, dass die kurzen Thesen zur Aktualität des reformatorischen Bildungsverständnisses im Sinne einer Richtungsangabe zu lesen sind und nicht erschöpfend gemeint sein können. Statt weiterer Ausführungen und Erklärungen dazu wende ich mich am Ende dieses Beitrags gleichwohl neuen Herausforderungen zu, die mir für die Zukunftsbedeutung dieses Bildungsverständnisses besonders wichtig erscheinen.

\section{Evangelisches Bildungsdenken vor neuen Herausforderungen}

Schon zu Beginn habe ich darauf aufmerksam gemacht, dass es in meinem Beitrag nicht um eine museale Betrachtung gehen soll, die sich im Geschichtlichen erschöpft oder die gar ganz auf eine bloße Verehrung der Reformation hinauslaufen würde. Von einem aktiv wahrgenommenen Erbe kann nur gesprochen werden, wo dieses angeeignet und dann auch konstruktiv weiterentwickelt wird. Diese Absicht betrifft nicht nur die Form, sondern gilt auch in inhaltlicher Hinsicht. Es ist nicht davon auszugehen, dass das reformatorische Bildungserbe Lösungen für sämtliche Bildungsfragen unserer Gegenwart bieten könnte. Besonders bei den nun aufzunehmenden neuen Herausforderungen kann dies noch einmal deutlich werden. Dabei konzentriere ich mich auf drei solche Herausforderungen für die Zukunft, die wiederum nur in knapper Form umrissen werden.

Die erste neue Herausforderung betrifft das Verhältnis zur Erziehungswissenschaft. Eine der ausgesprochenen Stärken der protestantischen Bildungstradition wird zu Recht darin gesehen, dass diese Tradition eine große Offenheit für die Erziehungswissenschaft aufweist, eben weil Erziehung reformatorisch auch unter weltlichen Aspekten thematisiert wird. Unter dieser Voraussetzung konnte die in der Vergangenheit in Deutschland immer wieder besonders enge Verbindung zwischen Pädagogik und Protestantismus allererst entstehen und produktiv ausgestaltet werden, indem pädagogische Perspektiven kirchlich integriert wurden und evangelisch-theologische Sichtweisen pädagogisch zum Tragen kommen konnten. Heute hingegen findet eine konfessionell oder religiös gebundene Pädagogik weithin keine erziehungswissenschaftliche Zustimmung mehr. Aus der Pädagogik ist die Erziehungswissenschaft geworden, die sich selbst, auch angesichts eines religiös und weltanschaulich pluralen Handlungsfeldes wie der Schule, religiös neutral verstehen will. Wenn das evangelische Bildungsdenken sich mit der in dieser Weise autonom verstehenden Erziehungswissenschaft neu ins Verhältnis setzen will, wird sie auch neue Formen des interdisziplinären Dialogs finden müssen, weil die Berufung auf die pädagogisch fruchtbare reformatorische Bildungstradition allein nicht mehr

${ }^{28}$ Vgl. dazu aus psychologisch-pädagogischer Sicht R.L. Fetz, K.H. Reich, P. Valentin, Weltbildentwicklung und Schöpfungsverständnis. Eine strukturgenetische Untersuchung bei Kindern und Jugendlichen, Stuttgart, W. Kohlhammer, 2001. 
überzeugt. Bis heute ist es aber nur sehr punktuell gelungen, zeitgemäße, für beide Seiten attraktive Dialogmöglichkeiten zwischen Erziehungswissenschaft und Theologie zu entwickeln - was sich inzwischen gerade angesichts der religiösen und weltanschaulichen Pluralität mit ihren Herausforderungen auf beide Seiten nachteilig auswirkt. Denn in einer solchen pluralen Situation braucht auch die Erziehungswissenschaft vermehrt religionsbezogene Kompetenzen, während Theologie und Kirche nicht ohne die Erziehungswissenschaft in öffentlich plausibler Form klären können, worin die Gegenwartsbedeutung des reformatorischen Bildungsverständnisses unter religiös und weltanschaulich veränderten Voraussetzungen bedeuten kann. Über das reformatorische Bildungserbe sowie die traditionelle Verbindung zwischen Pädagogik und Protestantismus hinaus sind in dieser Hinsicht komplexere Modelle zu entwickeln, die beispielsweise eine dialogische, auf verschiedene Religionen und Traditionen bezogene und insofern pluralistische Grundlegung der Erziehungswissenschaft statt einer lediglich wie auch immer neutralen Position ermöglichen.

Als zweite Herausforderung nenne ich das Erfordernis, das evangelische Bildungsdenken konsequent mit einer Verankerung in der Zivilgesellschaft zusammen zu denken. Diese Herausforderung hat mit dem Verhältnis zwischen Staat und Kirche sowie mit dem Selbstverständnis der evangelischen Kirche zu tun. Ihrem ganzen Selbstverständnis zufolge kann die evangelische Kirche auch nach der Trennung zwischen Kirche und Staat ihren Ort nicht einfach im Privatbereich finden, auf den sie mitunter verwiesen wird (Religion als reine Privatangelegenheit). Sie will in der Öffentlichkeit wirken, sich positionieren und engagieren sowie in einer auch öffentlich erkennbaren Weise gesellschaftliche Mitverantwortung übernehmen. Unter den Voraussetzungen eines demokratisch verfassten Gemeinwesens muss sie daher als Institution der Zivilgesellschaft ausgestaltet werden. ${ }^{29}$ Im Blick auf Bildung impliziert dies beispielsweise, dass die zivilgesellschaftliche Bedeutung kirchlicher Bildungsangebote verdeutlicht werden muss und dass entsprechende Neuakzentuierungen für solche Angebote erforderlich sind. Exemplarisch versucht wurde dies bislang vor allem für die Konfirmandenarbeit, die sich in ihrer gegenwärtigen Gestalt, zumindest in einer Reihe europäischer Kirchen und Länder, auch als Bildung für die Zivilgesellschaft verstehen lässt. Das gilt insbesondere für die in der Konfirmandenarbeit unterstützten prosozialen Werte sowie für die Einführung junger Menschen in Formen des ehrenamtlichen Engagements, das für die Konfirmandenarbeit inzwischen eine zentrale Rolle spielt, zumindest in einer Reihe von europäischen Ländern bzw. Kirchen. ${ }^{30}$

${ }^{29}$ Vgl. grundlegend dazu W. Huber, Kirche in der Zeitenwende. Gesellschaftlicher Wandel und Erneuerung der Kirche, Gütersloh, Bertelsmann, 1998.

${ }_{30}$ Vgl. dazu die Studien F. Schweitzer, G. Hardecker, C.M. Maßß, W. Ilg, K. Lißmann in Verbindung mit P. Schreiner, B. Sendler-Koschel, Jugendliche nach der Konfirmation. Glaube, Kirche und eigenes Engagement - eine Längsschnittstudie, Gütersloh, Gütersloher Verlagshaus, 2016; F. Schweitzer, T. Schlag, H. Simojoki, K. Tervo-Niemelä (eds.), Confirmation, Faith, and Volunteerism. A Longitudinal Study on Protestant Adolescents in the Transition Towards Adulthood. European Perspectives, Gütersloh, Gütersloher Verlagshaus 2017. 
Mit der dritten und letzten Herausforderung komme ich in anderer Weise noch einmal zurück auf die religiöse und weltanschauliche Pluralität, die vielleicht die größte neue Herausforderung für das evangelische Bildungsdenken darstellt. Denn hier geht es um eine Umstellung im epochalen Sinne. Die in der evangelischen Tradition vorgegebenen Leitbegriffe von Frieden und Gerechtigkeit als übergreifende Ziele für Bildung und Schule können zwar auch in diesem Falle zur Anwendung kommen, aber zu der Art und Weise, wie dies in interreligiösen Zusammenhängen geschehen soll, finden wir in der Tradition noch kein direktes Vorbild. Denn nicht nur beim Bildungsdenken, sondern auch insgesamt haben die evangelische Kirche und Theologie sehr lange gebraucht, ehe sie sich überhaupt auf Fragen zum Verhältnis zwischen Christentum und anderen Religionen oder Weltanschauungen eingelassen haben. ${ }^{31}$

Aus heutiger Sicht geht es um eine "Toleranz aus Glauben". ${ }^{32}$ So gesehen muss Toleranz dem Christentum oder auch anderen Religionen nicht einfach von außen aufgenötigt werden - eine mitunter im Verhältnis zum Islam eingesetzte Strategie, die sich aber als kontraproduktiv erwiesen hat, da sie nur Widerstände auslöst. Innere Haltungen können nicht von außen erzwungen werden. Stattdessen ist nach den religiösen Voraussetzungen der Toleranz zu fragen, wie sie in verschiedenen christlichen und nicht-christlichen Traditionen zu finden sind. In der reformatorischen Tradition ist dabei vor allem an das Verständnis von Rechtfertigung zu denken. Denn dieses Verständnis schließt ein, dass der Mensch auch über seinen eigenen Glauben nicht verfügen kann. Der Glaube ist in reformatorischer Sicht gerade kein menschliches Werk, sondern eine Gabe Gottes, die den Menschen erst zur Gewissheit im Glauben kommen lässt. Für das evangelische Bildungsdenken erwächst daraus die Aufgabe, eine solche "Toleranz aus Glauben" auch pädagogisch wirksam werden zu lassen. Ich selbst vertrete daher die Auffassung, dass Pluralitätsfähigkeit zu einem zentralen Bildungsziel geworden ist. ${ }^{33}$ Gemeint ist damit die Fähigkeit, sich weder fundamentalistisch gegen die religiöse und weltanschauliche Vielfalt zu stellen noch sich relativistisch allem lediglich anzupassen. Stattdessen anzustreben ist eine prinzipienorientierte, immer auch theologisch begründete religiöse Urteilsfähigkeit.

Ich schließe mit der These, dass das reformatorische Bildungserbe seine Bedeutung auch heute nicht verloren hat. Es steht für Frieden und Gerechtigkeit sowie, das füge ich hinzu: für Toleranz. Damit erinnert es Bildung und Schule auch heute an ihren Sinn. In konzentriertester Form lässt sich dies auch so ausdrücken:

${ }^{31}$ Vgl. dazu die Darstellung bei F. Schweitzer, Interreligiöse Bildung. Religiöse Vielfalt als Herausforderung und Chance, Gütersloh, Gütersloher Verlagshaus, 2014.

${ }^{32}$ Vgl. zu dieser Perspektive aus systematisch-theologischer Sicht C. Schwöbel, Toleranz aus Glauben. Identität und Toleranz im Horizont religiöser Wahrheitsgewissheiten, in: ders., Christlicher Glaube im Pluralismus. Studien zu einer Theologie der Kultur, Tübingen, Mohr Siebeck, 2003, 217-244.

33 Vgl. Schweitzer, Interreligiöse Bildung. Vgl. als kirchliche Stellungnahme auch Evangelische Kirche in Deutschland, Religiöse Orientierung gewinnen. Evangelischer Religionsunterricht als Beitrag zu einer pluralitätsfähigen Schule. Eine Denkschrift des Rates der EKD, Gütersloh, Gütersloher Verlagshaus, 2014. 
Frieden, Gerechtigkeit und Leben sowie Keine Bildung ohne Glaube - kein Glaube ohne Bildung.

\section{Kopsavilkums}

Rakstā tiek skaidrotas reformācijas un izglītības attiecības, īpaši pievēršoties protestantu mantojumam, kuru autors identificē un apspriež vēsturiskā un teorētiskā perspektīvāa. Raksta pirmajā daļā ir analizēts Martina Lutera viedoklis par izglītību, akcentējot religisko izglìtību skolā, kā arī katehētisko mācību baznīcāa. Raksta otrajā daļā iztirzātas vairākas perspektīvas, kas varētu būt nozīmīgas mūsdienu izpratnei par izglītību ne tikai pašā protestantismā, bet arī plašākā sabiedrībā un izglìtības filozofijā. Šìs perspektīvas attiecas, piemēram, uz attiecībām starp ticību vai religiju un izglītìbu, attiecībām starp ticību un zināšanām vai izglītības antropologiju. Nobeigumāa autors atklāj vairākus jaunus izaicinājumus, ar kuriem mūsdienu pasaule ir jāsastopas, lai izprastu protestantu izglītību. Galvenā problēma, kam protestantu tradīcijās nav pietiekami pievērsta uzman̄̄ba, ir dažādu reliǵiju un pasaules uzskatu plurālisms, kas raksturīgs mūsdienu sabiedrībai. Autors mēgina pierādīt, ka ir rodamas atbildes uz šiem izaicinājumiem atbilstoši protestantu tradīcijai un š̀ tradīcija ir gatava pieņemt arī jaunu lietu izpratni.

Atslēgvārdi: reformācija, izglìtība, izglītības vēsture, izglìtības filozofija.

Friedrich Schweitzer, Dr. rer. soc., Dr. theol. h.c., is professor for practical theology / religious education at the Protestant Faculty of Theology of the University of Tübingen in Germany. He is the author of many monographs and articles in several languages. His academic interests include religious education and general education, history of religious education, empirical research, interreligious education.

E-mail: Friedrich. Scvhweitzer@uni-tuebingen.de

Address: Evangelisch-Theologische Fakultät, Liebermeisterstr. 12, 72076 Tübingen, Germany 